Our Nature (2010) 8: 247-253

\title{
Diversity of Birds in Ousteri Wetland, Puducherry, India
}

\author{
A. Padmavathy ${ }^{*}$, R. Alexandar and M. Anbarashan \\ Department of Ecology and Environmental Sciences, Pondicherry University, Kalapet- 605014, Puducherry, \\ India \\ *E-mail: ecopadma@gmail.com
}

Received: 07.09.2010, Accepted: 13.11.2010

\begin{abstract}
Wetlands are very fragile and constitute treasure of biodiversity. The avifaunal diversity in Ousteri freshwater wetland, Puducherry, India, was studied to form a baseline data about several local and migratory bird species of this wetland. Birds are surveyed by direct sighting, calls and variable width line-transect method was carried out in December, 2009 to January, 2010. It resulted in total of 109 species of both migratory and local birds. Such unique diverse systems are under threats like reduction in water retention in this pond in summer, weed infestation; variations in food availability in different seasons, hunting, poaching and threat of predation on the breeding activity of birds affected the avifauna diversity in the study area.
\end{abstract}

Key words: Birds, divers ity, threats, conservation

\section{Introduction}

Avifaunas are important for the ecosystem as they play various roles as scavengers, pollinators, predators of insect pest, bioindicators of different kind of environment like urbanization and industrialization (Sharma, 1982; Bhattacharjee and Hazarika, 1985) human disturbance (Talukdar, 1997; Chakravarty, 1981) illumination (Sandhu and Dang, 1980). They are very sensitive indicators of pollution problems and function as early warning system (Gole, 1984; Becker, 2003).

Hussain et al. (1984) studied on Chilka lake in Orissa, Singh and Roy (1990) studied the ecology of birds of Kawar lake in Bihar, Sanjay (1993) studied about the ecology of birds at Kokkare-Bellur, Hosetti et al. (2001) researched on Ornithoecological aspects on Gudavi bird Sanctuary and recently Inac et al. (2008) studied the bird species of Kumasir lake. The above avifaunal studies impress upon the need for the inventory of avifaunal diversity of other such habitats especially in terms of conservation and management aspects.

Ousteri wetland was one of the important rainfed fresh water wetland of Puducherry, it's been declared as significant wetlands of Asia among the other 93 wetlands by Asian Wetland Bureau and IUCN (1988). The lake also been declared as a wildlife sanctuary by Government of Puducherry. In terms of biodiversity documentation, no studies had been done so far in this wetland ecosystem. The major aim of this study is to give a baseline data about several local and migratory bird species of the wetland.

\section{Materials and methods}

Puducherry located on the Coramandal coast between $11^{\circ} 52^{\prime}$ to $11^{\circ} 59^{\prime} \mathrm{N}$ and $79^{\circ} 45^{\prime}$ 
A. Padmayathy, R. Alexander and M. Anbarashan / Our Nature (2010) 8: 247-253

to $79^{\circ} 52^{\prime} \mathrm{E}$ and covers an area of $480 \mathrm{~km}^{2}$. It is limited on the east by the Bay of Bengal and on the other three sides by the Cuddalore and Villupuram districts of Tamil $\mathrm{Nadu}$ state. Ousteri lake was located in towards north at a distance of $10 \mathrm{~km}$ (Puducherry to Villupuram via Thirukanur). Wetland (Fig. 1) covers an area of about 175 ha (a part in Tamil Nadu and Puducherry) with wide range of aquatic

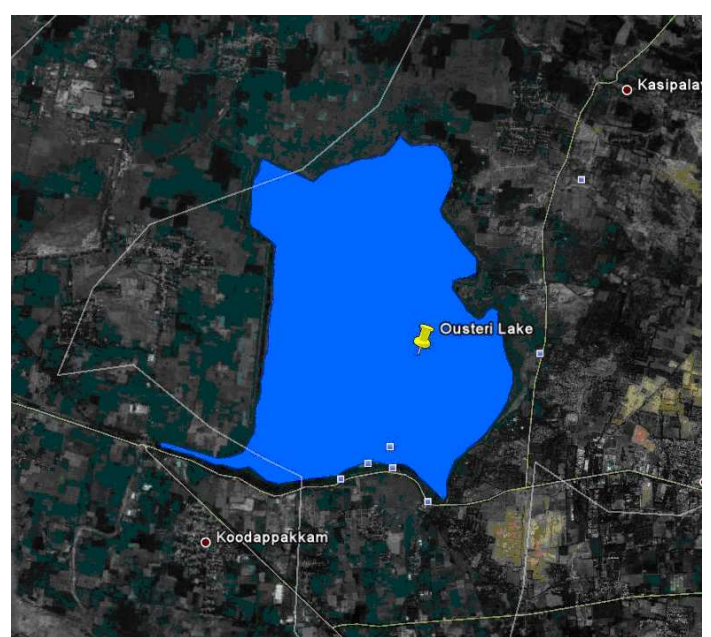

Figure 1. Map showing the Ousteri wetland, Puducherry, India

species and acts as a bird sanctuary. The vegetation ranges from small herbs to trees, which supports more migratory avifauna during their breeding season. Thus how it became an important tourist spot leading to more and more damage to such ecologically healthy system. Presently, this bird sanctuary supports a rich biodiversity in all aspects but lacks proper documentation. Such information was essential as the wetland serves as an important tourist spot apart from it as study area for some schools, college students and for many nature lovers and for conservation activities.
The study area experiences mean annual temperature of $30^{\circ} \mathrm{C}$ and mean annual rainfall about 1311-1172 $\mathrm{mm}$. The mean number of annual rainy days is 55 , the mean monthly temperature ranges from $21.3^{\circ}-30.2^{\circ} \mathrm{C}$. The climate is tropical dissymmetric with the bulk of the rainfall during northeast monsoon OctoberDecember (Indian Meteorological Department, Chennai).

Field surveys were carried out systematically during December, 2009 to January, 2010 by direct sighting, calls and variable width line-transect method (Bibby et al., 2000; Sutherland, 2006) by walking along transects laid appropriately on the study area. The transects also traverse nearby agriculture land, sugarcane field, scrub jungle around the wetland. Opportunistic surveys in the nearby villages were also done to document a comprehensive checklist of birds of the area. The birds sighted during the period includes both water birds or and land birds. First phase of the bird survey is done during the major breeding season of birds, especially for the migratory birds, this data are the baseline can be used later in seasonal avifaunal diversity monitoring and their management.

\section{Results and discussion}

A total of 109 species of birds belonging to 96 genera and 46 families were recorded during the study (Table 1 and 2). Dominant families are Phylloscopidae and Accipitridae with 10 species followed by Pelecanidae with 7 species and 21 families represented by single species. As per the Red Data Book of IUCN, Pelecanus philippensis, Mycteria leucocephala, Numenius arquata and Sterna acuticauda 
A. Padmayathy, R. Alexander and M. Anbarashan / Our Nature (2010) 8: 247-253

Table 1-Check list of Avian fauna of Ousteri wetland, Puducherry, India

\begin{tabular}{|c|c|c|}
\hline Family & Common name & Zoological name \\
\hline Podicipedidae & Little grebe or Dabchick & Podiceps ruficollis Pallas, 1764 \\
\hline \multirow{7}{*}{ Pelecanidae } & Grey pelical or Spottedbilled & Pelecanus philippensis Gmelin,1789 \\
\hline & Little cormorant & Phalacrocorax niger Vieillot, 1817 \\
\hline & Cattle egret & Bubulcus ibis Linnaeus, 1758 \\
\hline & Grey heron & Ardea cinerea Linnaeus, 1758 \\
\hline & Great egret & Ardea alba Linnaeus, 1758 \\
\hline & Little erget & Egretta garzetta Linnaeus, 1766 \\
\hline & Paddy bird or pond heron & Ardeola grayii Sykes, 1832 \\
\hline \multirow[t]{3}{*}{ Ciconiidae } & Asian Openbill stork & Anastomus oscitans Boddaert, 1783 \\
\hline & Painted stork & Mycteria leucocephala Pennant, 1769 \\
\hline & White strok & Ciconia ciconia Linnaeus, 1758 \\
\hline \multirow[t]{2}{*}{ Threskiornituidae } & Spoonbill & Platalea leucorodia Linnaeus, 1758 \\
\hline & White ibis & Threskiornis aethiopicus Latham, 1790 \\
\hline Phoenicopteridae & Greater flamingo & Phoenicopterus roseus Pallas, 1811 \\
\hline Anhimiodae & Spotbill or Grey duck & Anas poecilorhyncha Forster, 1781 \\
\hline \multirow{10}{*}{ Accipitridae } & Blackwinged kite & Elanus caeruleus Desfontaines, 1789 \\
\hline & Brahmini kite & Haliastur indus Boddaert, 1783 \\
\hline & Common parish kite & Milvus migrans Boddaert, 1783 \\
\hline & Kestrel & Faiko tinnumculus Linnaeus, 1758 \\
\hline & Marsh harrier & Circus aeruginosus Linnaeus, 1758 \\
\hline & Montagu's harrier & Circus pygargus Linnaeus, 1758 \\
\hline & Shikra & Accipiter badius Gmelin, 1788 \\
\hline & Sparrow hawk & Accipiter nisus Linnaeus, 1758 \\
\hline & White scavengervulture or Pharao's chicken & Neophron percnopteru Linnaeus, 1758 \\
\hline & Changeable hawk eagle & Nisaetus cirrhatus Gmelin, 1788 \\
\hline Phasianidae & Grey partridge & Francolinus pondicerianus Gmelin,1789 \\
\hline \multirow[t]{5}{*}{ Charadriidae } & Common snipe or Fantial snipe & Gallinago gallinago Linnaeus, 1758 \\
\hline & Little ringed plover & Charadrius dubius Scopoli,1786 \\
\hline & Little stint & Calidris minuta Leisler, 1812 \\
\hline & Redwattled lapwing & Vanellus indicus Boddaert, 1783 \\
\hline & Yellow wattled lapwing & Vanellus malabaricus Boddaert, 1783 \\
\hline \multirow[t]{5}{*}{ Scolopaciade } & Common Sandpiper & Actitis hypoleucos Linnaeus, 1758 \\
\hline & Curlew & Numenius arquata Linnaeus, 1758 \\
\hline & Green sandpiper & Tringa ochropus Linnaeus, 1758 \\
\hline & Marsh sandpiper & Tringa stagnatilis Bechstein, 1803 \\
\hline & Wood sandpiper or Spotted sandpiper & Tringa glareola Linnaeus, 1758 \\
\hline Recurvirostriadae & Blackwinged stilit & Himantopus himantopus Linnaeus, 1758 \\
\hline \multirow[t]{3}{*}{ Laridae } & Blackbellied tern & Sterna acuticauda Gray, 1832 \\
\hline & Indian river tern & Sterna aurantia Gray $_{2} 1831$ \\
\hline & Indian whiskered tern & Chlidonias hybrida Pallas, 1811 \\
\hline \multirow[t]{3}{*}{ Columbidae } & Blue rock pigeon & Columba livia Gmelin, 1789 \\
\hline & Ring dove & Streptopelia capicola Sundevall, 1857 \\
\hline & Spotted dove & Streptopelia chinensis Scopoli, 1768 \\
\hline Psttacidae & Roseringed parakeet & Psittacula Krameri Linnaeus, 1766 \\
\hline \multirow[t]{3}{*}{ Cuculiadae } & Common hawk Cockoo or Brain feverbird & Cuculus varius Scopoli, 1786 \\
\hline & Koel & Eudynamys scolopacea Horsfield, 1840 \\
\hline & Pied crested cuckoo & Clamator jacobinus Franklin, 1831 \\
\hline Tytonidae & Barn owl & Tyto alba Bonaparte, 1850 \\
\hline Strigidae & Eagle owl or Indian greathorned owl & Bubo bubo Linnaeus, 1758 \\
\hline
\end{tabular}


A. Padmayathy, R. Alexander and M. Anbarashan / Our Nature (2010) 8: 247-253

\begin{tabular}{|c|c|c|}
\hline & $\begin{array}{l}\text { Screech owl } \\
\text { Spotted owl }\end{array}$ & $\begin{array}{l}\text { Otus asio Linnaeus, } 1758 \\
\text { Athene brama Temminck, } 1821\end{array}$ \\
\hline Caprimulfisae & Common Indian nightjar & Caprimulgus asiaticus Latham, 1790 \\
\hline Apodidae & $\begin{array}{l}\text { House swift } \\
\text { Palm swift }\end{array}$ & $\begin{array}{l}\text { Apus affinis Gray, } 1830 \\
\text { Cypsiurus parvus Lichtenstein, } 1823\end{array}$ \\
\hline Alcedinidae & $\begin{array}{l}\text { Pied kingfisher } \\
\text { Small blue kingfisher } \\
\text { White breasted kingfisher }\end{array}$ & $\begin{array}{l}\text { Ceryle rudis Linnaeus, } 1758 \\
\text { Alcedo atthis Linnaeus, } 1758 \\
\text { Halcyon smyrnensis Linnaeus, } 1758\end{array}$ \\
\hline Meropidae & $\begin{array}{l}\text { Small green bee bee-eater } \\
\text { Blueril bee bee-eater }\end{array}$ & $\begin{array}{l}\text { Merops oriantalis Latham, } 1802 \\
\text { Merops philippinus Linnaeus, } 1766\end{array}$ \\
\hline Coracidae & Indian roller or Blue jay & Coracias benghalensis Linnaeus, 1758 \\
\hline Upupisae & Hoopoe & Upupa epops Linnaeus, 1758 \\
\hline Capitonidae & Crinmsonbreasted barbet or Coppersmith & Megalaima haemacephala Muller,1776 \\
\hline Picidae & Goldenbacked woodpecker & Dinopium benghalense Linnaeus, 1758 \\
\hline Pittidae & Indian pitta & Pitta brachyura Linnaeus, 1766 \\
\hline Alaudidae & $\begin{array}{l}\text { Ashycroun finch lark } \\
\text { Bush lark } \\
\text { Indian small skylark }\end{array}$ & $\begin{array}{l}\text { Eremopterix grisea Scopoli,1786 } \\
\text { Mirafra assamica Horsfield,1840 } \\
\text { Alauda gulgula Franklin, } 1831 \\
\end{array}$ \\
\hline Hirundinidae & $\begin{array}{l}\text { Asian hous martin } \\
\text { Common swallo }\end{array}$ & $\begin{array}{l}\text { Delichon dasypus Bonaparte, } 1850 \\
\text { Hirundo rustica Linnaeus, } 1758\end{array}$ \\
\hline Motacillidae & $\begin{array}{l}\text { Indian tree pipit } \\
\text { Largepied wagtail } \\
\text { Paddyfield pipit or Indian pipit } \\
\text { Yellow wagtail }\end{array}$ & $\begin{array}{l}\text { Anthus hodgsoni } \text { Richmond,1907 } \\
\text { Motacilla maderaspatensis Gmelin, } 1789 \\
\text { Anthus novaeseelandiae Gmelin, } 1789 \\
\text { Motacilla flava Linnaeus, } 1758 \\
\end{array}$ \\
\hline Campephagidae & $\begin{array}{l}\text { Blackheaded cuckooshrike } \\
\text { Large cuckoo shrine } \\
\text { Small minicet }\end{array}$ & $\begin{array}{l}\text { Coracina melanoptera Rüppell,1839 } \\
\text { Coracina novaehollandiae Gmelin, } 1789 \\
\text { Pericrocotus cinnamomeus Linnaeus, } 1766\end{array}$ \\
\hline Picnonpidae & Redevented bulbul & Pycnonotus cafer Linnaeus, 1766 \\
\hline Irwnidae & Iora & Aegithina tiphia Linnaeus, 1758 \\
\hline Laniidae & $\begin{array}{l}\text { Brown shrike } \\
\text { Common wood shrine } \\
\text { Gren shrine }\end{array}$ & $\begin{array}{l}\text { Lanius cristatus Linnaeus, } 1758 \\
\text { Tephrodornis pondicerianus Gmelin, } 1789 \\
\text { Lanius excubitor Linnaeus, } 1758\end{array}$ \\
\hline Sylviidae & Blyth's reed warbler & Acrocephalus dumetorum Blyth,1849 \\
\hline Muscicapidae & Brown flycatcher & Muscicapa dauurica Pallas, 1811 \\
\hline Phylloscopidae & $\begin{array}{l}\text { Brownlead warbler or Chiff chaff } \\
\text { Common babbler } \\
\text { Indian robin } \\
\text { Tawny-flanked Prinia } \\
\text { Lesserwhite throat } \\
\text { Magpie robin } \\
\text { Paradise flycatcher } \\
\text { Spotted babbler } \\
\text { Tailor bird } \\
\text { Whiteheaded babbler }\end{array}$ & $\begin{array}{l}\text { Phylloscopus collybita Vieillot, } 1817 \\
\text { Turdoides caudatus, Dumont, } 1823 \\
\text { Saxicoloides fulicata Linnaeus, } 1766 \\
\text { Prinia subflava Gmelin, } 1789 \\
\text { Sylvia curruca Linnaeus, } 1758 \\
\text { Copsychus saularis Linnaeus, } 1758 \\
\text { Terpsiphone paradisi Linnaeus, } 1758 \\
\text { Pellorneum ruficeps Swainson, } 1832 \\
\text { Orthotomus sutorius Pennant, } 1769 \\
\text { Turdoides affinis Jerdon, } 1845\end{array}$ \\
\hline Dicaeidae & Tickell's Flowerpecker & Dicaeum erythrorhynchos Latham, 1790 \\
\hline Nectariniidae & $\begin{array}{l}\text { Little spider hunter } \\
\text { Purplerumped sunbird }\end{array}$ & $\begin{array}{l}\text { Arachnothera longirostra Latham, } 1790 \\
\text { Nectarinia zeylonica Linnaeus, } 1766\end{array}$ \\
\hline Prloceidae & $\begin{array}{l}\text { Purple sunbird } \\
\text { House sparrow }\end{array}$ & $\begin{array}{l}\text { Nectarinia asiatica Latham, } 1790 \\
\text { Passer domesticus } \text { Linnaeus, } 1758\end{array}$ \\
\hline Sturnidae & Brahimini muna or blackheaded myna & Sturnus pagodarum Gmelin, 1789 \\
\hline
\end{tabular}


A. Padmayathy, R. Alexander and M. Anbarashan / Our Nature (2010) 8: 247-253

\begin{tabular}{|c|c|c|}
\hline & Indian myna & Acridotheres tristis Linnaeus, 1766 \\
\hline Oriolisae & Golder oriole & Oriolus oriolus Linnaeus, 1758 \\
\hline Dicruridae & Fork-tailed Drongo & Dicrurus adsimilis Bechstein,1794 \\
\hline \multirow[t]{4}{*}{ Corvidae } & House crow & Corvus splendens Vieillot, 1817 \\
\hline & Jungle crow & Corvus macrorhynchos Wagler, 1827 \\
\hline & Ravens & Corvus corax Linnaeus, 1758 \\
\hline & Tree pie & Dendrocitta vagabunda Latham, 1790 \\
\hline Estrildidae & Black headed munia & Lonchura malacca Linnaeus, 1758 \\
\hline Rallidae & Eurasian hoot & Fulica atra Linnaeus, 1758 \\
\hline Pycnonotidae & White throated bulbul & Alophoixus flaveolus Gould,1836 \\
\hline
\end{tabular}

Table 2. List of total families with their respective genera and species number

\begin{tabular}{|c|c|c|}
\hline Family & Genera & Species \\
\hline Podicipedidae & 1 & 1 \\
\hline Pelecanidae & 6 & 7 \\
\hline Ciconiidae & 3 & 3 \\
\hline Threskiornituidae & 2 & 2 \\
\hline Phoenicopteridae & 1 & 1 \\
\hline Anhimiodae & 1 & 1 \\
\hline Accipitridae & 8 & 10 \\
\hline Phasianidae & 1 & 1 \\
\hline Charadriidae & 4 & 5 \\
\hline Scolopaciade & 3 & 5 \\
\hline Recurvirostriadae & 1 & 1 \\
\hline Laridae & 2 & 3 \\
\hline Columbidae & 2 & 3 \\
\hline Psttacidae & 1 & 1 \\
\hline Cuculiadae & 3 & 3 \\
\hline Tytonidae & 1 & 1 \\
\hline Strigidae & 3 & 3 \\
\hline Caprimulfisae & 1 & 1 \\
\hline Apodidae & 2 & 2 \\
\hline Alcedinidae & 3 & 3 \\
\hline Meropidae & 2 & 2 \\
\hline Coracidae & 1 & 1 \\
\hline Upupisae & 1 & 1 \\
\hline Capitonidae & 1 & 1 \\
\hline Picidae & 1 & 1 \\
\hline Pittidae & 1 & 1 \\
\hline Alaudidae & 3 & 3 \\
\hline Hirundinidae & 2 & 2 \\
\hline Motacillidae & 3 & 4 \\
\hline Campephagidae & 2 & 3 \\
\hline Picnonpidae & 1 & 1 \\
\hline Irwnidae & 1 & 1 \\
\hline Laniidae & 2 & 3 \\
\hline Sylviid ae & 1 & 1 \\
\hline Muscicapidae & 1 & 1 \\
\hline Phylloscopidae & 10 & 10 \\
\hline Dicaeidae & 1 & 1 \\
\hline
\end{tabular}


A. Padmayathy, R. Alexander and M. Anbarashan / Our Nature (2010) 8: 247-253

\begin{tabular}{lll} 
Nectariniidae & 2 & 3 \\
Prloceidae & 1 & 1 \\
Sturnidae & 2 & 1 \\
Oriolisae & 1 & 1 \\
Dicruridae & 1 & \\
Corvidae & 2 & 1 \\
Rallidae & 1 & 1 \\
Pycnonotidae & 1 & 1 \\
Estrildidae & 1 & \\
\hline
\end{tabular}

are near threatened species and Neophron percnopterus as endangered species, remaining all other species are found to be under least concern category were found during this survey.

This Ousteri wetland has been a main source of water for recharging the surrounding wells, bore wells, agriculture fields and industries around it. It is a vulnerable pond harboring plenty of resident and migratory birds. This wetland are under serious threats due to habitat loss, habitat degradation, paddy cultivation, sugarcane cultivation, casuarinas cultivation and extensive reed collection for thatching roofing, poaching birds, frequent reed fire and cutting trees are amongst threats from the locals. All this activities are resulting in loss of avifaunal diversity of the lake ecosystem (Alexandar, 2010).

\section{References}

Alexandar, R. 2010. Conservation of Ousteri lake. Correspondence, Curr. Sci. 98(4): 467.

Becker, P.H. 2003. Biomonitoring with bird. In Bioindicators and biomonitors, (Eds. B.A. Markert, A.M. Breure and M.C. Zechmeister). Amsterdam: Elsevier Science Ltd. pp. 677736.

Bhattacharjee, P.C., and B.C. Hazarika 1985. Roosting sites and roosting birds at Gauhati Municipal area. In Second international symposium on life sciences. November, 14-16, 1985. NEHU Shillong.
Bibby, C., B.N. Burges and D.A. Hill 2000. Bird census techniques, $2^{\text {nd }}$ edn. Academic Press, London.

Chakravarty, A.K. 1981. Effects of human interference on waterfowl of pools in Bangalore (Karnataka), India. In Symposium on Tropical Ecology, (Eds. R.S. Ambasht and H.N. Pandey). Silver Jubliee, October 5-10, 1981, Bhopal. pp. 37-38

Gole, P. 1984. Birds of a polluted river. J. Bomb. Nat. Hist. Soc. 81: 613-625.

Hosetti, B.B., B.C. Somanath and K.L. Naik 2001. Eco-ornithological studies on Gudavi Bird Sanctuary, Shimoga, Karnataka, India, cited. In Trends in wild life biodiversity conservation and management (Eds. B.B. Hosetti and M. Venkateshwarulu). vol. 1. Daya Publishing House, Delhi. pp. 269-289.

Hussain, S.A., K.K. Mohapatra and S. Ali 1984. Avifaunal profile of Chilka lake, a case for conservation. J. Bomb. Nat. Hist. Soc., Bombay, Technical report-4

Inac, S., O. Gorucu and A.H. Pinar 2008. The bird species of Kumasir lake (KahramanmarasTurkey) and a view of environmental ethics on sustainable wetland management. J. Envir. Biol. 29: 411-414.

IUCN 1988. Directory of Asian wetlands. ICBP and IWRB. 482 p.

Sandhu, P.S. and H.R. Dang 1980. Roosting behaviour of parakeets in relation to human disturbance. In Second all India Symposium on life sciences, March 9-11, 1980. Institute of Science, Nagpur.

Sanjay, G.S. 1993. An ecological study of birds at Kokkare Bellur. WWF-India, New Delhi. Final Report 
A. Padmayathy, R. Alexander and M. Anbarashan / Our Nature (2010) 8: 247-253

Sharma, I.K. 1982. Adverse effects of air, water and soil pollutions on flora and fauna of towns and villages of Western Rajasthan. In Symposium on environment consciousness, problems of pollution and conservation in Rajasthan. October 1-3, 1982.

Singh, J.P. and S.P. Roy 1990. Some aspects of ecology of birds of Kawar lake, Bihar.
J.Fresh Water Biol. 2: 175-189.

Sutherland, J.W. 2006. Ecological Census techniques, $a$ hand book. $2^{\text {nd }}$ edition, Cambridge University Press. 321 p.

Talukdar, B.K. 1997. Waterbirds of Dibru-saikhowa wild life sanctuary. Assam J. Nat. Cons. 9(2): 243-250. 